\title{
Preparation and Evaluation of Nanoemulgels Containing a Combination of Grape Seed Oil and Anisotriazine as Sunscreen
}

\author{
Anayanti Arianto ${ }^{1,2 *}$, Desi Yet Lie ${ }^{1}$, Sumaiyah Sumaiyah ${ }^{1,2}$, Hakim Bangun ${ }^{1,2}$ \\ ${ }^{1}$ Department of Pharmaceutical Technology, Faculty of Pharmacy, Universitas Sumatera Utara, Medan 20155, Indonesia; \\ ${ }^{2}$ Nanomedicine Center of Innovation, Universitas Sumatera Utara, Medan 20155, Indonesia
}

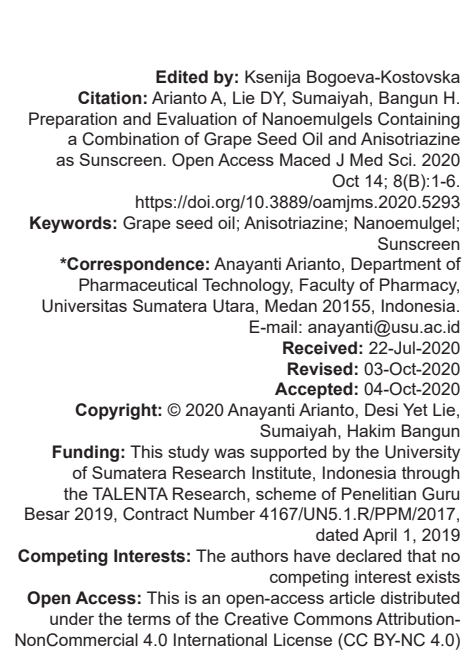

\section{Introduction}

The ultraviolet (UV) radiation (UVR) caused sunburn damage, photoaging, and skin cancer [1], [2], [3]. UVR can produce free radical oxygen species and damage the DNA of the skin. The deleterious effects of UVR are inhibited through the use of sunscreens [4]. Some chemical UV filters showed the adverse effects of sunscreen product such as irritant, allergic, phototoxic, and photoallergic [5]. Therefore, this research used grape seed oil to increase safety of sunscreen product. The use of grape seed oil (vegetable oil) could be a promising strategy to improve the SPF of sunscreen formulation with a reduction in content of anisotriazine. The sunscreen with high sun protection factor (SPF) can be obtained with nanotechnology formulations and combinations of sunscreen [6].

In this study, grape seed oil is used as natural UVB absorbent and combined with anisotriazine as a chemical UVA and UVB absorbent. Anisotriazine is an oil-soluble material and photostable. The solubility of the lipophilic ingredient of anisotriazine is improved by nanoemulsion formulation [7], [8].

Grape seed oil is a yellowish oil and odorless and rich in antioxidant compounds such as tocopherols (Vitamin E), tocotrienols, flavonoids, phenolic acids, and carotenoids so it is very well used in cosmetic formulations [9]. Antioxidants can provide a protective effect on the skin against damaging effect UVR mediated free radicals [10]. Oxidation of cellular biomolecules caused by acute exposure of human skin could be prevented by antioxidant, acting as free radical scavenger [3]. Vitamin $\mathrm{E}$ also absorbs strongly in the UVB region at wavelengths of 280-320 nm [11].

The SPF value of the sunscreen and physical stability can be increased using nanotechnology. Nanoemulsion has droplet size of 20-500 nm smaller than conventional emulsion that had droplet size of $0.1-$ $100 \mu \mathrm{m}$, so nanoemulsion more stable than emulsion. Nanoemulgel known as hydrogel-based nanoemulsion, with the presence of a thickening agent, the stability 
of the nanoemulgel preparation will be better due to a decrease in interface tension and an increase in viscosity and adhesion at the time of topical administration. Furthermore, nanoemulgel shows the benefits of being non-greasy, easily spread, and clean [12]. Thus, the aim of this research was to formulate and evaluate physical stability and determined SPF value of sunscreen nanoemulgels containing a combination of grape seed oil and anisotriazine by a simple and reliable UV-visible spectrophotometry.

\section{Materials and Methods}

The materials used in this study were grape seed oil (Aceites Borges Pont, S.A.U., Spanyol), anisotriazine (Ashland, America), Tween 80, and sorbitol which were purchased from PT. Bratachem, Medan, methylparaben, propylparaben, Span 80, propylene glycol, CMC Na, glycerol, ethanol, Carbopol 940, and triethanolamine (TEA) were purchased from CV. Rudang Jaya, Medan. These materials were analytical grade.

The nanoemulsions containing grape seed oil were prepared with surfactants (Tween 80) and cosurfactants (sorbitol) using the high-energy emulsification method [13], [14]. In this study, the preparation of nanoemulsions using magnetic stirrer and ultrasonicator. The aqueous phase was prepared by dissolving preservative (methylparaben and propylparaben) in water and Tween 80 was added to this solution. The mixture of grape seed oil and sorbitol was mixed to the water phase and stirred at $2400 \mathrm{rpm}$ for $6 \mathrm{~h}$ by 79-1 laboratory magnetic stirrer with heater (China). Then, the nanoemulsions were sonicated for $1 \mathrm{~h}$ by Ultrasonic Cleaner Branson 1510 E-MT (USA) until transparent nanoemulsions were produced. The optimum nanoemulsion formulation was selected based on the smallest droplet size used for the preparation of nanoemulgel containing $5 \%$ grape seed oil and anisotriazine 2 and $4 \%$.

The 2\% Carbopol 940 solution was used to prepared the nanoemulgels containing grape seed oil and anisotriazine. First, Carbopol 940 was dispersed in pure water and added with TEA to $\mathrm{pH} 6-6.5$ to obtain the gel base of $2 \%$ Carbopol 940 solution. Nanoemulgels were prepared by mixing the obtained nanoemulsions containing grape see oil and anisotriazine with the gel base of $2 \%$ Carbopol 940 (ratio of nanoemulsion and gel $4: 1$ ), then stirred using a magnetic stirrer with heater at 2400 rpm for 8 hours and ultrasonicated until a transparent nanoemulgels were produced.

The preparation of emulsion containing $4 \%$ grape seed oil and $3.2 \%$ anisotriazine was prepared by dissolving anisotriazine and Span 80 in grape seed oil and then heated to $70^{\circ} \mathrm{C}$. The preservatives (methyl and propyl parabens) were dissolved in propylene glycol and glycerol and added CMC Na solution and this mixture was heated to $70^{\circ} \mathrm{C}$. After that, the mixture of anisotriazine, Span 80, and grape seed oil was added to the mixture of preservative and CMC Na solution and stir with magnetic stirrer for 45 min [15]. Emulgel was obtained by mixing the obtained emulsions with a gel base of $2 \%$ of Carbopol 940 (ratio of emulsion and gel 4:1) with gentle stirring for $10 \mathrm{~min}$ [16].

The mean droplet size for nanoemulgel formulations was measured by laser light scattering using the Nanoparticle Analyzer HORIBA SZ-100, Germany. The $\mathrm{pH}$ measurement is done using $1 \%$ nanoemulgel solution in pure water with a digital $\mathrm{pH}$ meter (Hanna instrument).Viscosity measurement was carried out using the Brookfield DF-E Viscometer and the sunscreen nanoemulgel was measured every 2 weeks for 12 weeks of storage at room temperature.

Physical stability evaluation of nanoemulgels was done by storing its at $28 \pm 2{ }^{\circ} \mathrm{C}$ (room temperature), $40 \pm 2^{\circ} \mathrm{C}$ (high temperature), and $4 \pm 2^{\circ} \mathrm{C}$ (low temperature) for 12 weeks. Nanoemulgel formulas were observed through visual inspection for their color, odor, and phase separation with observation every week [16], [17].

Cycling test for sunscreen nanoemulgels and emulgel was done by putting them in the freezer at $4 \pm 2{ }^{\circ} \mathrm{C}$ for $24 \mathrm{~h}$ and then put in Climatic Chamber Memmert, Germany, at $40 \pm 2^{\circ} \mathrm{C}$ for $24 \mathrm{~h}$ and repeated in 6 cycles. After that, the physical stability was observed. Centrifugation test was done using centrifuges (Hitachi CF $16 \mathrm{R} \times$ II, Japan) with a rotation of $3750 \mathrm{rpm}$ during for $5 \mathrm{~h}$ at $25 \pm 2{ }^{\circ} \mathrm{C}$. The physical stability of the nanoemulgel was observed [17].

The morphology and globule size of nanoemulgel containing $4 \%$ grape seed oil and $3.2 \%$ anisotriazine were analyzed using transmission electron microscope (JEOL JEM 1400, Japan).

The sunscreen nanoemulgel and emulgel for SPF determination were diluted to volume with $96 \%$ ethanol to the final concentration of the sample which was $200 \mu \mathrm{g}$ per $\mathrm{mL}$. The absorption spectra were obtained in the range of 290-320 nm with an interval 5 $\mathrm{nm}$ measurement using a spectrophotometer UV-visible (Shimadzu UV 1800, Japan) and six determinations were made for each sample [18].

The SPF value was calculated using the following equation:

$$
\mathrm{SPF}=\mathrm{CF} \times \sum_{290}^{320} \times \mathrm{EE}(\lambda) \times \mathrm{l}(\lambda) \times \operatorname{Abs}(\lambda)
$$

Where: CF: Correction factor $(=10)$; EE: Erythemal effect spectrum; I: Solar intensity spectrum; 
Abs: Absorbance of sample. The absorbance value obtained is multiplied by EE $\mathrm{x}$ I for each interval [19].

\section{Results}

The nanoemulsions were prepared in three formulations using a ratio variation of Tween 80 as surfactant and sorbitol as cosurfactant is presented in Table 1.

Table 1: Composition of nanoemulsions containing grape seed oil

\begin{tabular}{llll}
\hline Ingredients & Quantity of $100 \mathrm{~mL}(\%)$ & & \\
\cline { 2 - 4 } & $\mathrm{F} 1$ & 5 & $\mathrm{~F} 3$ \\
\hline Grape seed oil & 5 & 26 & 5 \\
Tween 80 & 25 & 34 & 33 \\
Sorbitol & 35 & 0.1 & 0.1 \\
Methylparaben & 0.1 & 0.02 & 0.02 \\
Propylparaben & 0.02 & 100 & 100 \\
Distilled water to & 100 & \\
\hline
\end{tabular}

The sunscreen nanoemulsion formulations (F1, F2, and F3) showed a light yellow color and transparency, as shown in Figure 1. Formulation of F3 that used the highest Tween 80 (surfactant) shows the lowest of droplet size $(9.2 \mathrm{~nm})$ and this formula was selected for preparation nanoemulsion and nanoemulgel containing combination of grape seed oil and anisotriazine.

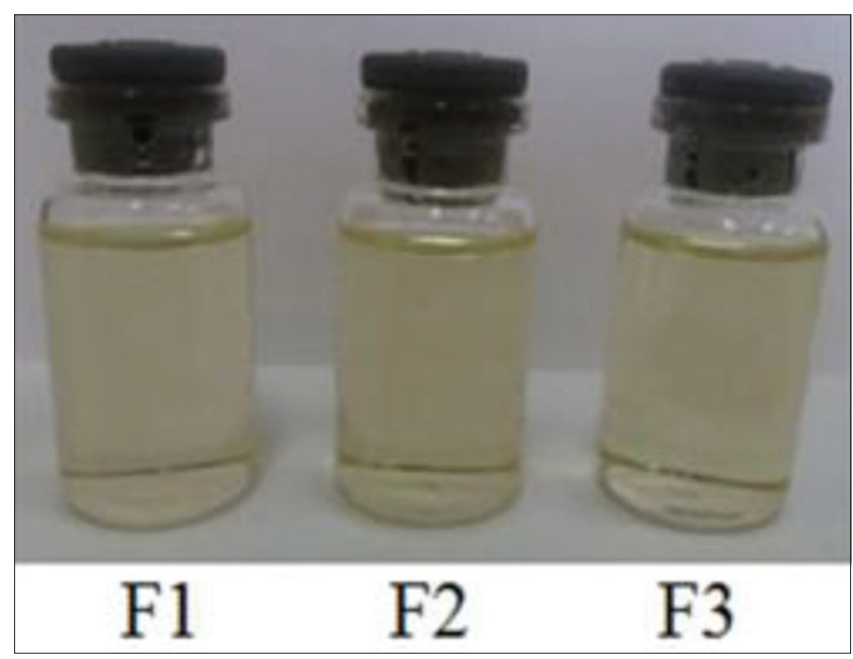

Figure 1: Formulation of nanoemulsion containing 5\% grape seed oil with a ratio variation of Tween 80 and sorbitol F1 (25:35), F2 (26:34), F3 (27:33)

The composition of nanoemulgels containing grape seed oil and anisotriazine is presented in Table 2. All sunscreen nanoemulgel formulations (F4, F5, and F6) had a transparent appearance, slightly yellow in color and stable during storage for 12 weeks under varied temperature condition, as shown in Figure 2.

The composition of emulgel containing grape seed oil and anisotriazine is presented in Table 3. The result of emulgel formulation was milky white. The droplet size of this emulgel formulation was larger than nanoemulgel, therefore, the appearance of emulgel was not transparent. The physical stability evaluation of
Table 2: Composition of grape seed oil and anisotriazine nanoemulgels

\begin{tabular}{llll}
\hline Ingredients (\% wt/wt) & \multicolumn{2}{l}{ Quantity of $100 \mathrm{~mL}(\%)$} & $\mathrm{F} 6$ \\
\cline { 2 - 4 } (Nanoemusion) & $\mathrm{F} 4$ & 5 & 5 \\
\hline Grape seed oil & 5 & 2 & 4 \\
Anisotriazine & 0 & 27 & 27 \\
Tween 80 & 27 & 33 & 33 \\
Sorbitol & 33 & 0.10 & 0.10 \\
Methylparaben & 0.10 & 0.02 & 0.02 \\
Propylparaben & 0.02 & 100 & 100 \\
Distilled water to & 100 & & \\
Ingredient (\% wt/wt) (Gel base) & & & \\
$\quad$ Carbopol 940 & 2 & & \\
$\quad$ TEA & 1 & & \\
$\quad$ Distilled water to & $100 \mathrm{~mL}$ & &
\end{tabular}

nanoemulgel containing grape seed oil and anisotriazine showed that the nanoemulgel was stable, there was no phase separation, while the emulgel formulation showed the phase separation after storage for 7 weeks at room temperature as it is shown in Figure 3. It can be explained that nanoemulsions prepared using a magnetic stirrer at $2400 \mathrm{rpm}$ and ultrasonicator was found to be more stable for longer duration of time when compared to emulsions prepared by mechanical agitation [20].

Table 3: Composition of grape seed oil and anisotriazine emulgel

\begin{tabular}{ll}
\hline Ingredients (emulsion) & Quantity of $100 \mathrm{~mL}(\%)$ \\
\hline Grape seed oil & 5 \\
Anisotriazine & 4 \\
Tween 80 & 1.25 \\
Span 80 & 3.73 \\
Methylparaben & 0.10 \\
Propylparaben & 0.02 \\
Propylene glycol & 8 \\
Glycerin & 10.4 \\
CMC Na & 0.8 \\
Distilled water to & 100 \\
Ingredients gel base & \\
Carbopol 940 & 2 \\
TEA & 1 \\
Distilled water to & $100 \mathrm{~mL}$ \\
\hline TEA Triethanolamine &
\end{tabular}

The $\mathrm{pH}$ of the nanoemulgel and emulgel containing combination of grape seed oil and anisotriazine decreased during the storage of 12 weeks as it is shown in Table 4, but the $\mathrm{pH}$ values were within the range of $\mathrm{pH}$ skin, normal from 4.5 to 6.0 , which are considerably acceptable and less irritable for use in human skin [21].

Table 4: $\mathrm{pH}$ of nanoemulgel and emulgel at storage for 12 weeks at room temperature

\begin{tabular}{lllll}
\hline Week & Average $\mathrm{pH}$ & & \\
\cline { 2 - 5 } & $\mathrm{F} 4$ & $\mathrm{~F} 5$ & $\mathrm{~F} 6$ & Emulgel \\
\hline 0 & $7.03 \pm 0.00$ & $7.06 \pm 0.11$ & $7.20 \pm 0.10$ & $7.03 \pm 0.06$ \\
2 & $7.00 \pm 0.00$ & $7.03 \pm 0.06$ & $7.00 \pm 0.00$ & $6.96 \pm 0.06$ \\
4 & $6.96 \pm 0.06$ & $6.93 \pm 0.06$ & $6.93 \pm 0.06$ & $6.86 \pm 0.06$ \\
6 & $6.86 \pm 0.06$ & $6.83 \pm 0.06$ & $6.83 \pm 0.06$ & $6.73 \pm 0.06$ \\
8 & $6.66 \pm 0.06$ & $6.60 \pm 0.10$ & $6.56 \pm 0.11$ & $6.43 \pm 0.05$ \\
10 & $6.46 \pm 0.06$ & $6.40 \pm 0.10$ & $6.36 \pm 0.06$ & $6.10 \pm 0.10$ \\
12 & $6.23 \pm 0.06$ & $6.20 \pm 0.10$ & $6.13 \pm 0,06$ & $5.76 \pm 0.06$ \\
\hline
\end{tabular}

The droplet size of all nanoemulgels $<200 \mathrm{~nm}$ was increased after storage for 12 weeks at room temperature, but the size of droplets was still smaller than $500 \mathrm{~nm}$, as shown in Figure 4. The nanoemulsion is obtained when the size of droplet reaches approximately 20-500 nm [22]. Otherwise, the droplet size of the emulgel was larger than nanoemulgel. 


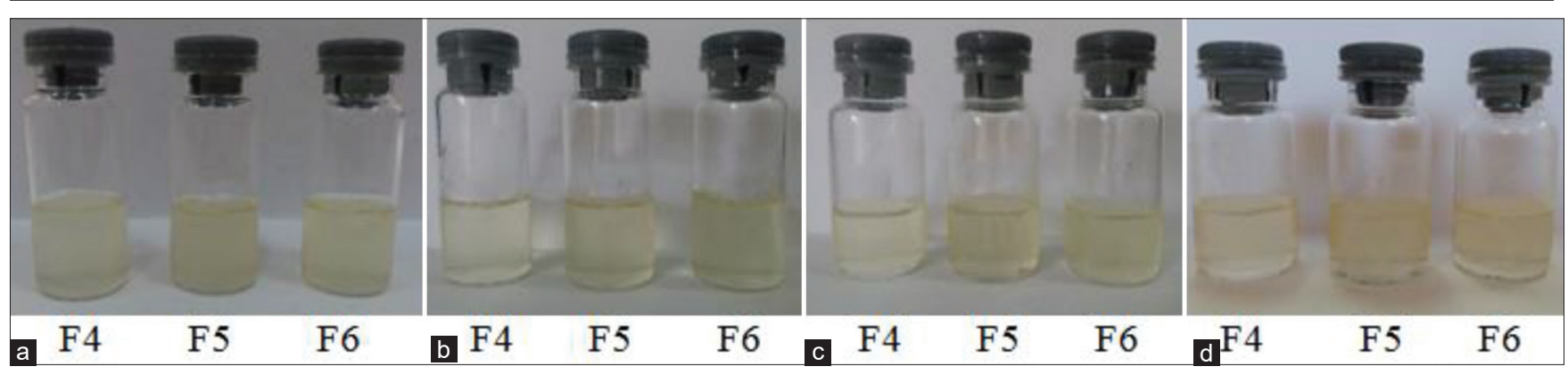

Figure 2: Formulation of sunscreen nanoemulgel (F4: Containing 4\% grape seed oil only, F5: Containing 4\% grape seed oil and 1.6\% anisotriazine, F6: Containing 4\% grape seed oil and 3.2\% anisotriazine), (a) before storage, (b) after storage for 12 weeks at room temperature, (c) after storage for 12 weeks at high temperature, (d) after storage for 12 weeks at low temperature

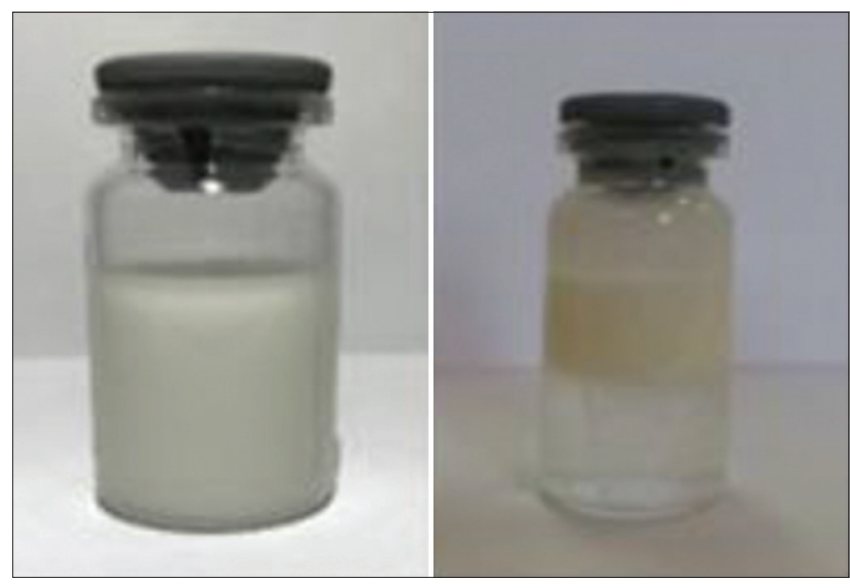

Figure 3: Formulation of sunscreen emulgel containing $4 \%$ grape seed oil and 3.2\% anisotriazine (a: Before storage, b: After storage for 7 weeks at room temperature)

Therefore, the emulgel was not physically stable during 12 weeks storage at room temperature.

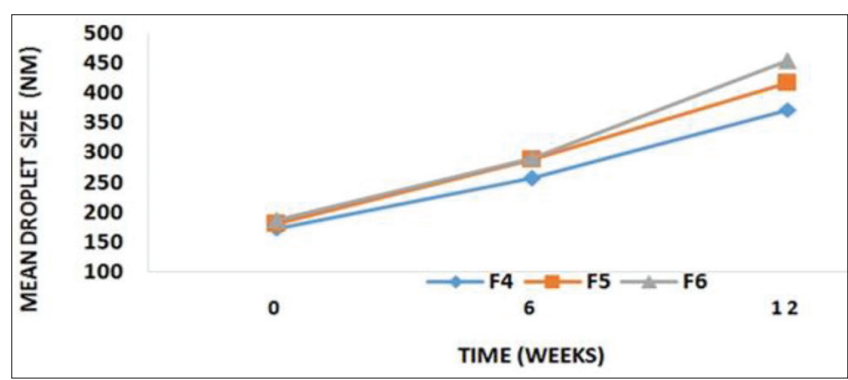

Figure 4: Droplet size of sunscreen nanaoemulgels (F4, F5, and F6)

All nanoemulgel preparations (F4, F5, and F6) remained stable after centrifugation test with no sedimentation and creaming or phase separation observed in Figure 5, but the emulsion was not stable with the formation of phase separation as it is shown in Figure 6. The centrifugation test was performed to determine the effect of gravity on the stability of the nanoemulgel which is equivalent to the gravitational force for a year.

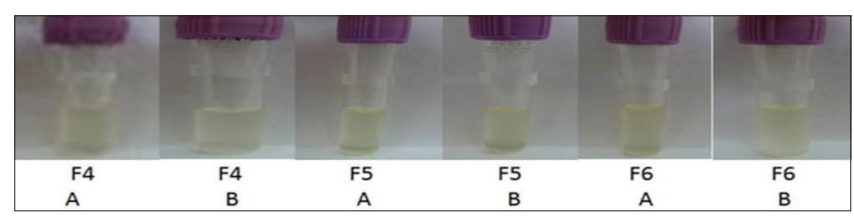

Figure 5: Appearance of sunscreen nanoemugel formulations after centrifugation

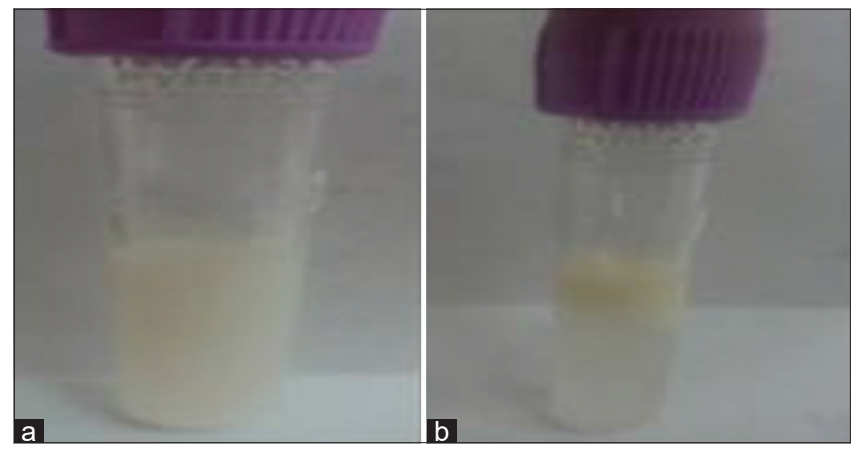

Figure 6: Appearance of emulgel formulation containing 4\% grape seed oil and 3.2\% anisotriazine after centrifugation test (a: Before centrifugation, $b$ : After centrifugation)

The viscosity of the nanoemulgel formulations (F4, F5, and F6) showed an increase in viscosity value for 12 weeks of storage, while the emulgel showed a decrease in viscosity value as it is shown in Figure 7. This is because the increasing of the droplet size of the nanoemulgel causing an increase in the viscosity of the preparations during storage. While, the viscosity on the emulgel during 12 weeks of storage decreased, this is associated with phase separation.

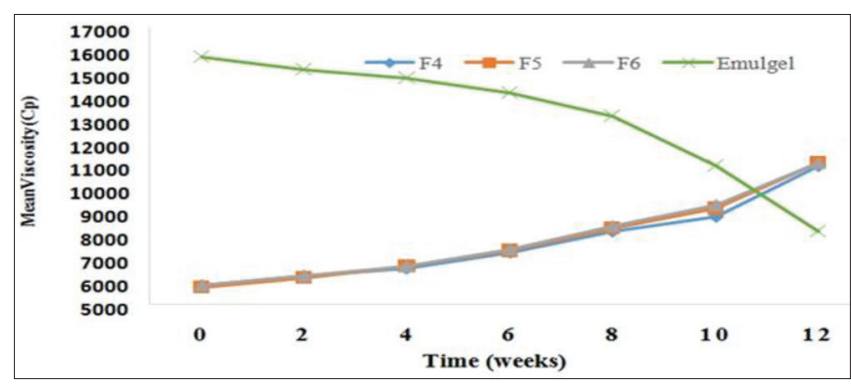

Figure 7: Effects of storage time on viscosity of sunscreen nanoemugels and emugel

There was no sign of phase separation for any sunscreen nanoemulgel formulations after the cycling test, indicating that the nanoemulgel would be stable under test conditions as is presented in Figure 8.

The average SPF value of all formulations of sunscreen nanoemulgels is shown in Table 5. It indicates that the average SPF value of nanoemulgels containing combination of grape seed oil and anisotriazine (F5 and F6) is higher than those containing grape seed oil only (F4). 


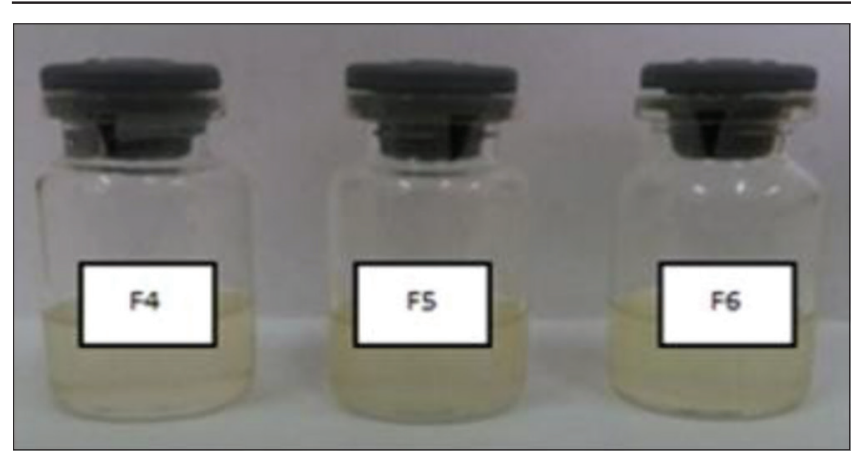

Figure 8: Cycling test result of sunscreen nanoemugels (F4, F5, and F6)

The transmission electron microscopy image of nanoemulgel is shown in Figure 9. The morphological analysis results of nanoemulgel were spherical in size $<200 \mathrm{~nm}$ without any aggregation. This evaluation was performed on nanoemulgel containing $4 \%$ grape seed oil and anisotriazine $3.2 \%$ (F6) with the highest SPF value among all sunscreen nanoemulgel formulations.

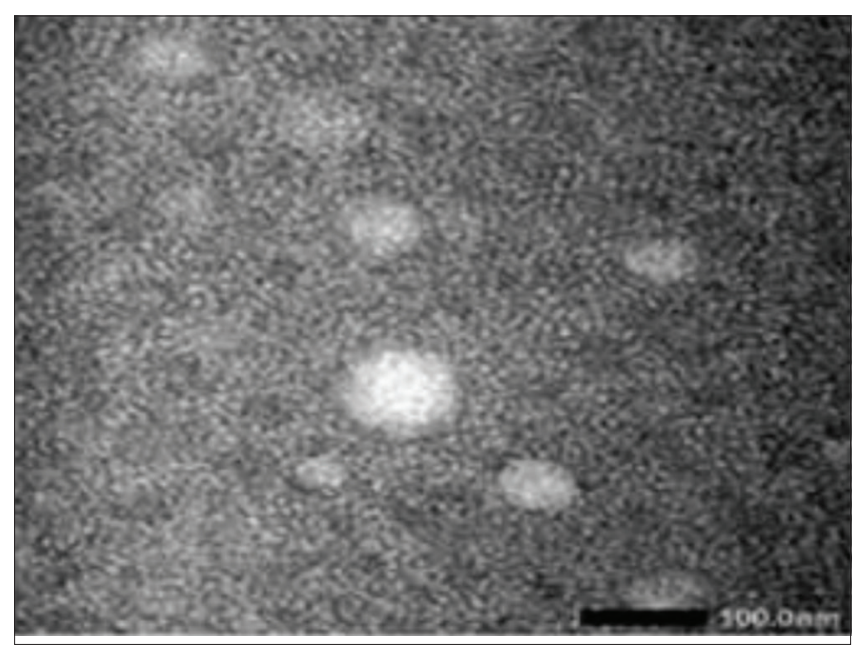

Figure 9: TEM image of sunscreen nanoemulgel containing 4\% grape seed oil and $3.2 \%$ anisotriazine (F6)

Table 5: Average SPF value of sunscreen nanoemulgel and emulgel

\begin{tabular}{|c|c|c|c|c|c|c|c|}
\hline \multirow[t]{2}{*}{ Formula } & \multicolumn{6}{|c|}{ SPF value } & \multirow{2}{*}{$\begin{array}{l}\text { Average SPF } \\
\text { value }\end{array}$} \\
\hline & 1 & II & III & IV & $\mathrm{V}$ & $\mathrm{VI}$ & \\
\hline$\overline{F 4}$ (nanoemulgel) & 11.315 & 11.291 & 11.195 & 11.072 & 11.078 & 11.068 & $11.169 \pm 0.113$ \\
\hline F5 (nanoemulgel) & 16.127 & 16.378 & 16.191 & 16.156 & 16.098 & 16.149 & $16.183 \pm 0.100$ \\
\hline F6 (nanoemulgel) & 19.495 & 19.644 & 19.484 & 19.244 & 19.086 & 19.001 & $19.325 \pm 0.232$ \\
\hline Emulgel & 11.615 & 11.804 & 11.946 & 11.997 & 12.006 & 12.110 & $11.913 \pm 0.161$ \\
\hline
\end{tabular}

\section{Discussion}

The nanoemulsion in this study was prepared using Tween 80 as a surfactant with an HLB value of 15 ( $>10)$ and sorbitol as cosurfactant to form an oil/ water nanoemulsion [8]. Nanoemulsion with smaller droplet size was achieved using the magnetic stirrer and ultrasonicator. In the preparation of nanoemulsions, ultrasonication is used to produce mechanical vibrations and cavitation for the formation of stable nanoemulsion with small droplets [13]. Nanoemulsion containing grape seed oil and variations of anisotriazine is formulated by adding different concentrations of anisotriazine $(2,4$, and $6 \%)$. A precipitate formation observed in the highest concentrations (6\%) of anisotriazine, thus, the nanoemulsions with 2 and $4 \%$ were selected for the preparation of nanoemulgel, in which there was no precipitation formation after 12 weeks from the preparation time.

Nanoemulgel with transparent appearance is obtained by adding a gel solution to nanoemulsion in a ratio of $4: 1$. The mean droplet size of all nanoemulgel formulations was $<200 \mathrm{~nm}$. Nanoemulgel containing $4 \%$ grape seed oil and $3.2 \%$ anisotriazine (F6) had a droplet size of $187.5 \mathrm{~nm}$. The droplet size of nanoemulgel in the range of $20-200 \mathrm{~nm}$ could be obtained using magnetic stirrer and ultrasonicator, which leads to a substantial reduction of the droplet size. The cost of this equipment was cheaper than other high-energy equipment and more flexible on surfactant selection than low-energy emulsification method [23], [24]. The physical stability evaluation results of the nanoemulgel which showed more stable compared to emulgel, this can be explained that nanoemulgel prepared with highenergy emulsification which results in a smaller droplet size but the emulgel prepared by mechanical agitation which results in a larger droplet size [20].

A combination of grape seed oil and anisotriazine provides higher SPF values due to the synergistic effect of the combination in the formulation of sunscreen nanoemulgel. In addition, nanoemulgel has a smaller particle size so that it will absorb more UVB rays which results in a higher SPF value. This results appropriate with previous research that a high SPF sunscreen product could be obtained by nanotechnology [6].

\section{Conclusion}

The sunscreen nanoemulgel formulation containing $4 \%$ grape seed oil and $3.2 \%$ anisotriazine was more stable compared with emulgel formulation. The SPF value of this nanoemulgel formulation is higher than nanoemulgel formulation without anisotriazine and emulgel.

\section{References}

1. Parkin DM, Mesher D, Sasieni P. 13. Cancers attributable to sola (ultraviolet) radiation exposure in the UK in 2010. Br J Cancer. 2011;105(2):S66-9. $\quad$ https://doi.org/10.1038/bjc.2011.486 PMid:22158324 
2. D'Orazio J, Jarrett S, Amaro-Ortiz A, Scott T. UV Radiation and the skin. Int J Mol Sci. 2013;14(6):1222248. https://doi.org/10.3390/ijms140612222 PMid:23749111

3. Pandel R, Poljšak B, Godic A, Dahmane R. Skin photoaging and the role of antioxidants in its prevention. ISRN Dermatol. 2013;2013:930164. https://doi.org/10.1155/2013/930164 PMid:24159392

4. Green AC, Williams GM, Logan V, Strutton GM Reduced melanoma after regular sunscreen use: Randomized trial follow-up. J Clin Oncol. 2011;29(3):25763. https://doi.org/10.1200/jco.2010.28.7078 PMid:21135266

5. HeurungAR,RajuSI,WarshawEM.Adversereactionstosunscreen agents: Epidemiology, responsible irritants and allergens, clinical characteristics, and management. Dermatitis. 2014;25(6):289$326 . \quad$ https://doi.org/10.1097/der.0000000000000079 PMid:25384223

6. Arianto A, Cella G, Bangun H. Preparation and evaluation of sunscreen nanoemulsions with synergistic efficacy on SPF by combination of soybean oil, avobenzone, and octyl methoxycinnamate. Open Access Maced J Med Sci. 2019;7(17):2751-6. https://doi.org/10.3889/oamjms.2019.745 PMid:31844431

7. Limsuwan T, Amnuikit T. Effect of grape seed extract in sunscreen lotion on sun protection factor (SPF) determined by in vitro method. In: Proceedings of the $6^{\text {th }}$ International Conference on Bioinformatics and Biomedical Science. United States: ACM Press; 2017. https://doi.org/10.1145/3121138.3121192

8. Debnath S, Satayanarayana KVG, Kumar GV. Nanoemulsion-a method to improve the solubility of lipophilic drugs. PHARMANEST Int J Adv Pharm Sci. 2011;2(2-3):72-83.

9. Shinagawa FB, de Santana FC, Torres LR, Mancini-Filho J. Grape seed oil: A potential functional food? Food Sci Technol. 2015;35(3):399-406. https://doi.org/10.1590/1678-457x.6826

10. Garavaglia J, Markoski MM, Oliveira A, Marcadenti A. Grape seed oil compounds: Biological and chemical actions for health. Nutr Metab Insights. 2016;9:59-64. https://doi.org/10.4137/nmi. s32910

11. Goswami PK, Mayuri S, Rashmi S. Natural sunscreen agents: A review. SAJP. 2013;2(6):458-63.

12. Basera K, Bhatt G, Kothiyal P, Gupta P. Nanoemulgel: A novel formulation approach for topical delivery of hydrophobic drugs. WJPPS. 2015;4(10):1872-6.

13. Delmas T, Piraux H, Couffin AC, Texier I, Vinet F, Poulin P, et al. How to prepare and stabilize very small nanoemulsions. Langmuir. 2011;27(5):1683-92. https://doi.org/10.1021/ la104221q
14. Koroleva MY, Yurtov EV. Nanoemulsions: The properties, methods of preparation and promising applications. Russ Chem Rev. 2012;81(1):21-43. https://doi.org/10.1070/ rc2012v081n01abeh004219

15. Estanqueiro M, Conceição J, Amaral MH, Santos D, Silva JB, Lobo JM. Characterization and stability studies of emulsion systems containing pumice. Braz J Pharm Sci. 2014;50(2):3619. https://doi.org/10.1590/s1984-82502014000200016

16. Jain A, Gautam SP, Gupta Y, Khambete H, Jain S. Development and characterization of ketoconazole emulgel for topical drug delivery. Der Pharm Sin. 2010;1(3):221-31.

17. Iradhati $\mathrm{AH}$, Jufri $\mathrm{M}$. Formulation and physical stability test of griseofulvin microemulsion gel. Int J Appl Pharm. 2017;9:23. https://doi.org/10.22159/ijap.2017.v9s1.22_27

18. Dutra EA, da Costa e Oliveira DA, Kedor-Hackmann ER Santoro MI. Determination of sun protection factor (SPF) of sunscreens by ultraviolet spectrophotometry. Rev Bras Ciên Farma. 2004;40(3):381-5. https://doi.org/10.1590/ s1516-93322004000300014

19. Sayre RM, Agin PP, LeVee GJ, Marlowe E. A comparison of in vivo and in vitro testing of sunscreening formulas. Photochem Photobiol. 1979;29(3):559-66. https://doi.org/10.1111/j.1751-1097.1979.tb07090.x PMid:441130

20. Shekar MC, Chary BB, Srinivas S, Kumar BR, Mahendrakar MD, Varma MV. Effect of ultrasonication on stability of oil in water emulsions. Int J Drug Deliv. 2011;3(1):133-40. https://doi. org/10.5138/ijdd.2010.0975.0215.03063

21. Chikakane $\mathrm{K}$, Takahashi $\mathrm{H}$. Measurement of skin $\mathrm{pH}$ and its significanceincutaneousdiseases. ClinDermatol.1995;13(4):299$306 . \quad$ https://doi.org/10.1016/0738-081x(95)00076-r PMid:8665438

22. Bernardi DS, Pereira TA, Maciel NR, Bortoloto J, Viera GS, Oliveira GC, et al. Formation and stability of oil-in-water nanoemulsions containing rice bran oil: In vitro and in vivo assessments. J Nanobiotechnol. 2011;9(1):44. $\quad$ https://doi.org/10.1186/1477-3155-9-44 PMid:21952107

23. Yukuyama MN, Ghisleni DD, Pinto TJ, BouChacra NA. Nanoemulsion: Process selection and application in cosmetics-a review. Int $\mathrm{J}$ Cosmet Sci. 2015;38(1):13-24. https://doi.org/10.1111/ics.12260 PMid:26171789

24. Mehmood T, Ahmed A, Ahmed Z, Ahmad MS. Optimization of soya lecithin and Tween 80 based novel Vitamin D nanoemulsions prepared by ultrasonication using response surface methodology. Food Chem. 2019;289:664-70. https:// doi.org/10.1016/j.foodchem.2019.03.112 